\title{
The Basis of Time in the Universe
}

\author{
Vincent Vesterby
}

\section{Introduction}

"What is time?" Lee Smolin asked in the preface to his book Time Reborn. He goes on to say "This deceptively simple question is the single most important problem facing science as we probe more deeply into the fundamentals of the universe. All of the mysteries physicists and cosmologists face-from the Big Bang to the future of the universe, from the puzzles of quantum physics to the unification of the forces and particles - come down to the nature of time" [1].

For thousands of years people have wondered about time. What is it? Why does it occur? Why does time have the specific qualities that it has, such as the distinction between the future, the present, and the past? It has not previously been possible to provide definitive, conclusive, answers to those questions because the qualities of time to which people have access do not take the mind all the way to the basis of time.

Analyzing the known qualities of time in order to work down to the basis of time is a topdown process. This method has always failed because the qualities of time, such as the evident "arrow" of time, the relentless progression of time, and the distinction between the past, the present, and the future, do not indicate the nature of the basis of time. The research described in this paper that revealed the basis of time in the universe was a bottom-up process.

The discovery of the intrinsic nature of time was not the goal of the research that lead to that discovery [2]. The goal of the research was transdisciplinary understanding aimed at furthering effective communication between the disciplines of science, and further, between the sciences and the humanities. Space and the continuing-existence of space are foundational to the universe - deeply foundational to all the disciplines. The goal was to develop an understanding of the intrinsic natures of space and its continuing-existence that was clear, precise - and real - that everyone could understand, and do so without discipline specific studies in science and mathematics.

The resulting description of the qualities of the continuing-existence of space was, amazingly, a description of time. The continuing-existence of space was analyzed and described before it was recognized as the basis of time- a bottom-up process of discovery.

The following part of the paper is divided into four major sections. The first section discusses methodology, and explains why is it is necessary to distinguish between concepts and the reality referents of concepts. This section also explains why the understanding of space and the understanding of the continuing-existence of space must 
be based on objective observation, without the inclusion of any form of speculation-no assumptions, hypotheses, or theories.

The two following major sections provide the necessary understanding of the intrinsic nature of space and the intrinsic nature of the continuing-existence of space.

The fourth major section discusses how the continuing-existence of space plays the role of time in the universe. This section provides understanding of the intrinsic natures of the present, the past, and the future, clarifying the actual, or ontological, relations between them.

\section{On method}

\section{Distinguishing between concepts and their reality-referents}

The discussion in this paper is not about concepts. It is about the reality-referents of concepts. It is not about the concept of time. It is about time itself. This paper is about the intrinsic nature of the reality-referents of concepts.

In this paper concepts are recognized to be mental tools that can be used by the mind to achieve understanding of the real-world, which for the most part, exists outside the mind, outside the brain. A significant difference between concepts and their reality-referents is that concepts, as mental entities, are confined within brains, while the reality-referents of concepts occur throughout the universe, including within brains for the reality-referents of some concepts. For example, the physical cellular neural pathways within a brain are the reality-referents for the concept of neural pathways.

Another significant difference is that concepts interrelate conceptually while their realityreferents interrelate physically. For example, concepts cannot push against each other, while material objects push against each other in causal interrelation.

A third difference is that within the mind concepts can be interrelated logically or illogically, rationally or irrationally. They can be interrelated in any pattern or jumble in which the mind associates them.

The mind does not have that ability with the reality-referents of the concepts. The relations between the reality-referents are determined by structural-logic- the manner in which the intrinsic qualities of things that exist determine the kinds of relations that can occur between those things. That is, relations between the reality-referents of concepts are determined by factors beyond the concepts themselves.

\section{Why understanding must be based on observation}

The relations between reality-referents of concepts are determined by factors intrinsic to the reality-referents themselves. That is why the understanding of relations between 
reality-referents must be based on understanding the intrinsic nature of the realityreferents. This requires both observation and the use of structural-logic.

The understanding of the intrinsic nature of space, and from that the understanding of the intrinsic nature the continuing-existence of space, and therefore of time, must be based on the observation of space.

\section{Observing space}

While the existence of space is independent of the existence of matter, the relation of matter to space and certain relations of matter, one part to another, can be used to notice the existence of space. Matter is not space. To look just at matter is to not look at space. While the nature of matter, its substantiality, does not reveal anything about the nature of space, matter occupies space, and there are aspects of that relation that give clues to the existence and nature of space.

It can be observed that:

- Matter occupies space.

- Matter moves through space.

- Groups of material objects exist in space as patterns-of-organization, with the distance and direction relations between the material components of the patterns the same as the distance and direction relations between the spatial-locations the material components occupy.

The prime imperative of analysis is to look to the situation under analysis, allowing the intrinsic qualities of that situation to dictate to the mind the qualities of the mind's understanding of the situation. To apply the prime imperative of analysis to space-look at the space the matter occupies, moves through, and forms patterns in.

Space does not reflect photons. Matter reflects photons. The visual sense has the capacity to detect where the photons are coming from, whether near or further away. There is always a space between the material source of the photons and the eye. There is a space there that the photons cross on their way to the eye. This space between photon source and visual receptors is evident - represented, depicted — in the mind's mental image that results from the visual process.

To understand what space is, it is necessary to focus the attention on that space between photon source and the eye.

Further, all of the matter that humans can see occurs in the form of units. Many of these material units are coherently bonded into larger units, but even then, it is all units. Observation of certain relations between these units can lead to the observation of space. 
When observing a group of material units that are not coherently bonded and that are not adjacent to one another, it is possible to observe the space between the units. For the investigation of the nature of space, the focus of observation is on this space between the material units, ignoring the units themselves.

\section{Understanding the intrinsic nature of space-what is it that is continuing to exist?}

To understand why the continuing-existence of space plays the roles of time in the universe - to understand why the occurrence of spatial continuing-existence establishes the qualities of time in the universe - it is necessary to understand the actual nature of the continuing-existence of space.

The continuing-existence of space is a consequence of the existence of space-space exists and it thereby continues to exist. Because spatial continuing-existence is a consequence of the existence of space, to understand the nature of spatial continuingexistence, it is necessary first to understand the nature of space itself, that is, to understand the nature of what it is that is continuing to exist.

The following sections (1 through 9) discuss certain qualities of space that are relevant to understanding why the continuing-existence of space plays the role of time in the universe.

\section{Space exists}

The existence of space can be detected visually. The space is there, it exists. It can also be detected by moving the hand through the space between a photon reflecting object and the eye, or by moving the hand in the space between objects. If the units are removed, the space is still there, independent of those units.

\section{Space is place}

Matter occupies space. Space is a place that matter can occupy. Space is a place where matter can exist.

Matter moves through space. Space is a place where matter can move-a place through which matter can move.

Groups of material objects exist in space as patterns-of-organization. Each component of the group occupies a place in space. The specific pattern-of-organization of the group is based on the distances and directions of the components from one another. The distances and directions of the components are the distances and directions between the spatialplaces occupied by the components. The distances and directions are qualities of the spatial-place occupied by the group of components. The pattern-of-organization of the group occupies an extended area of spatial-place. Space is place in which matter exists, 
moves, and forms patterns. Each factor-existence, motion, pattern-occurs because space is place.

\section{Space is three-dimensional}

It is not possible to exist with less than three dimensions of extension. In any situation, if one of the three dimensions of extension were to be reduced all the way such that there was no longer any extension in that dimension, there would not be anything left in the situation to have extension in the other two dimensions.

Three-dimensionality is one of the universal foundational existence-factors that are required for there to be existence-any form of existence. An existence-factor is any factor whose presence and roles are required for there to be existence. There are three universal foundational existence-factors that together are required for there to be existence: existential-quantity, three-dimensionality, and continuing-existence.

From any location in space, the three-dimensionality of spatial-place extends infinitely (See section 6 below) in every direction.

Space is not, and cannot be, extensionally four-dimensional because three-dimensional extension is all inclusive. There is no place for an extensional fourth dimension. Any hypothesis, theory, or mathematical system that proposes more than three dimensions of spatial-extension is not descriptive of anything that exists or that could exist in the real world.

\section{Space is immaterial}

Matter occupies spatial-place. Matter is substantial. The substantiality of matter occupies spatial-place.

When observed, space does not appear to display any attributes of substantiality. To say that space is substantial in any way is speculation. For substantial matter to occupy spatial-place, it is not required for space itself to be substantial. Substantial matter occupies nonsubstantial—immaterial—spatial-place.

\section{The immateriality of space precludes any interaction between space and anything else that exists.}

That which is immaterial lacks the capacity to interact with that which is substantial. Space cannot interact with matter. The presence of matter in space has no effect whatsoever on the spatial-place the matter occupies, or on the surrounding space.

When matter occupies space, the immateriality of the occupied space precludes any effect of the occupied space on the matter. 


\section{Space cannot be different from one place to another}

Because space is immaterial, spatial-place is intrinsically uniform in nature. Space lacks the capacity to be different from one place to another. Because space is immaterial it cannot move, curve, expand, or have any different qualities from part to part.

All parts of space are identical in what they are, differing only in their intrinsic-identities as different parts of space. Intrinsic-identity is what something is intrinsically-what something is in and of itself distinct from other things that exist and distinct from its relations to other things that exist.

\section{Space is infinite}

Space is place. Spatial-place is three-dimensional. Three-dimensional material objects occupy three-dimensional spatial-place. The three-dimensional extension of material objects occupies the three-dimensional extension of spatial-place.

The extension of substantial material objects is limited. Beyond the limit, edge, or surface of a material object there is space. There is space inside a material object - the space the object occupies. And there is space outside an object— the space all around it.

The situation is not the same with immaterial spatial-place. Immateriality does not have an observable or detectable limit, edge, or surface. The three-dimensional extension of immaterial spatial-place does not have an observable or detectable limit, edge, or surface.

It is possible to speculate about an edge or limit to the extension of space- - but that brings up a question. Beyond the limit of a material object there is space. What would be beyond an edge of immaterial place? There is no answer. Any answer would be baseless speculation.

Space is infinite due to what it is - three-dimensional extension of immaterial placewhich cannot be different in what it is one part from another. Since space is immaterial, it does not have the wherewithal to exist here and not exist there. Because space exists in the manner in which it does exist, it cannot not exist. Since space exists in the manner in which it exists, it cannot be other than infinite.

\section{All infinite space is coexistent}

Space is immaterial. Due to that immateriality, space does not have the attributes necessary to be different from one part to another, other than in their intrinsic-identities as different parts of space.

Local parts of space can be observed to be coexistent. Due to the immateriality of space, unobserved parts of space cannot be different from observed local parts of space. All parts of space are coexistent. All infinite space is coexistent. 


\section{Space constitutes an existential-context, a place in which to exist, for all else that exists}

All else that exists does so within space.

- To exist is to occupy spatial-place.

- Three-dimensional spatial-place constitutes an existential-context for the existentially required intrinsic three-dimensional aspect of everything else that exists.

All else that exists is existentially-dependent on space for a place to be.

- To not exist in space is to not exist at all.

Space, existing as the infinite three-dimensional extension of universally coexistent immaterial place, constitutes the foundational component of the universe.

\section{Understanding the intrinsic nature of the continuing-existence of space}

\section{Space not only exists - it continues to exist}

The continuing-existence of space can be observed by looking up at the night sky and watching it for a while. The observed space is there, and it continues to be there. It continues to exist, throughout the period of observation.

\section{Distinguishing the difference between the continuance of spatial extension and the continuance of spatial continuing-existence}

It is important to not confound the extension of space with the continuing-existence of space. Both the extension of spatial-place and the continuing-existence of space are forms of continuance. However, these two forms of continuance are distinct from one another. The continuance of the extension of spatial-place is static and can be measured by a ruler of some kind, while the continuance of spatial continuing-existence is a form of change and can be measured by a clock.

Understanding how the continuance of the extension of spatial-place is different from the continuance of the continuing-existence of space helps the mind to understand the actual nature of spatial continuing-existence. Understanding the nature of spatial continuingexistence enables the understanding of why spatial continuing-existence constitutes the basis of time in the universe. 


\section{Distinguishing between the coexistent-sequential-difference of spatial-extension, which is static, and the noncoexistent-sequential-difference of spatial continuing- existence, which is a form of ongoing change}

The extension of immaterial spatial-place is continuous. Anything that exists in a continuous manner has parts. For example, there is the part that is one half of the continuance and the other part that is the other half. Anything that exists in a continuous extensional manner has parts, such as the part that exists on one side of any particular point along that extension, and the part that exists on the other side of that point.

The parts are existentially distinct from one another, different in that they occur at different places along the extension. Because all parts of space are coexistent, the parts of the continuous extension of spatial-place are coexistent. The coexistent parts along the continuance of the extension occur sequentially - from any part along the extension there is an adjacent part, and beyond that there is another part, and so on.

The distinct, sequential, coexistent parts occur in a pattern of coexistent-sequentialdifference. Because immaterial space cannot move or change in any way, this pattern of coexistent-sequential-difference is static, unchanging.

Spatial continuing-existence, like spatial-extension, occurs in a continuous manner. And, as with spatial-extension, the continuance of spatial continuing-existence has parts.

If a person looks up at the nighttime sky and observes the continuing-existence of the star strewn space above, and while doing so holds a hand in a closed position, and then, while still observing the ongoing continuing-existence of that part of space, holds the hand in an open position, it becomes immediately evident that the two parts of the continuingexistence of space were distinct - existentially different.

During the part of the continuing-existence of space in which the hand was closed, the part of the spatial continuing-existence in which the hand was open did not then exist.

Then, during the part of the spatial continuing existence in which the hand was open, the prior part during which the hand was closed was no longer there, it had ceased to exist.

These two different parts of spatial continuing-existence were not coexistent. They occurred sequentially, one after the other, in a noncoexistent manner-noncoexistentsequential-difference.

- While the parts of the extension of spatial-place are coexistent, the parts of spatial continuing-existence are not coexistent.

- While the coexistent-sequential-difference of spatial-extension is static, without any form of change, the noncoexistent-sequential-difference of spatial continuingexistence occurs as a form of continuously ongoing change. 
- While static coexistent-sequential-difference is measured with a ruler, changing noncoexistent-sequential-difference is measured with a clock.

Understanding the various roles of sequential-difference in the universe is significant because:

- It helps distinguish between unchanging situations (coexistent-sequentialdifference) and changing situations (noncoexistent-sequential-difference).

- It provides deep understanding of change because all forms of change occur as noncoexistent-sequential-difference.

- Despite the differences in the various forms of change (continuing-existence, motion, the process of emergence, and developed processes such as metabolism, sustainable systems, plate tectonics, and solar systems), understanding the roles of sequential-difference provides a way to understand the relations of the different forms of change to each other.

\section{The continuing-existence of space is the foundational source of new existence in the universe}

Other than the parts being coexistent in the one and noncoexistent in the other, there is something more that distinguishes static unchanging coexistent-sequential-difference from changing noncoexistent-sequential-difference.

With the coexistent-sequential-difference of spatial-extension, everything always stays the same. Nothing ever changes. There is never anything new.

With the noncoexistent-sequential-difference of spatial continuing-existence, the situation is never the same. There is constant change. Spatial continuing-existence initiates newness.

With the noncoexistent-sequential-difference of spatial continuing-existence, only the current part of the continuing-existence of space exists. The prior part of spatial continuing-existence no longer exists. And the future part of the ongoing continuingexistence of space has not yet occurred.

The part of the continuing-existence of space that is occurring currently is noncoexistently distinct from the part of that continuing-existence that occurred just previous. The current part of spatial continuing-existence did not yet exist when the previous part was occurring. Now, as the current part exists, it is newly existent. As space continues to exist, there is continuously new part of that ongoing continuance - new part of ongoing noncoexistent-sequential-difference of spatial continuing-existence.

The occurrence of change is based on the occurrence of newness. Newness is required for there to be change. Change is the occurrence of newness. 


\section{New part of spatial continuing-existence is universally concurrent}

- Because space is infinite, the occurrence of spatial continuing-existence is infinite.

- Because space is infinitely omnipresent, the occurrence of spatial continuingexistence is infinitely omnipresent.

- Because all space is coexistent throughout its infinite occurrence, spatial continuing-existence occurs concurrently throughout - the current part of spatial continuing-existence is the same throughout infinite space.

- Because all space is coexistent, and because all space continues to exist concurrently the occurrence of new part of spatial continuing-existence is simultaneous throughout infinite space.

\section{The continuing-existence of space is unidirectional}

The continuing-existence of space is the continuance of space being there. Continuance of being there is, by nature, one way-intrinsically unidirectional.

The continuing-existence of space is an ongoing sequence of change. The continuingexistence of space continuously, progressively, initiates the occurrence of new part of that continuance, and thereby progresses unidirectionally in that manner. This can be observed and measured with a clock.

Sequentially continuous new part of continuing-existence continuously replaces the current part. With the sequential progressive occurrence of new part of continuingexistence, the current part sequentially ceases to exist. The direction of continuingexistence occurs with the sequentially progressive occurrence of new part. With continuing-existence, what exists unidirectionally initiates what follows.

\section{The continuing-existence of space is nondimensional.}

Space is immaterial. The continuing-existence of space has an immaterial mode-ofbeing - an immaterial manner in which it occurs. That which is immaterial cannot have intrinsic units that are distinct from one another in structure or process. An immaterial mode-of-being can have parts, but not units. The ongoing change of continuingexistence, being continuous without units, does not have any length-just continuously occurring change. The occurrence of change itself, having no length, is nondimensional.

\section{The continuing-existence of space constitutes an existential-context, a place in which to occur, for all other forms of change}

\section{Continuing-existence is a form of change}

Because space is the foundational component of the universe, spatial continuingexistence is the foundational form of continuing-existence, (and thereby the foundational 
form of change). There are two other secondary but still fundamental forms of continuing-existence. Both matter and motion have intrinsic continuing-existence.

Matter, like space, is there, it exists, and like space, matter continues to be there, it continues to exist.

As a motion is continuing to occur - as the matter continues to pass through space - that motion is continuing to exist. There is, then, the continuing-existence of the motion itself - the duration of the ongoing motion as measured by a clock.

Continuing-existence is one of the universal foundational existence-factors that are required for there to be existence. These three are the fundamental forms of continuingexistence.

- The continuing-existence of immaterial space.

- The continuing-existence of substantial matter.

- The continuing-existence of motion.

\section{The three forms of continuing-existence are isomorphies.}

An isomorphy is a pattern-of-organization that occurs in two to many different situations or systems [3]. An isomorphic pattern-of-organization can occur in space, in material structure, and in material process. Isomorphies play roles in the origins, existence, organization, and changes within the situations or systems in which they occur.

The continuing-existence of space, of matter, and of motion, are all forms of continuingexistence, and thus all occur as ongoing noncoexistent-sequential-difference with the continuous initiation of new part. They differ in what it is that is continuing to exists, and in their existential-dependency relations with one another.

\section{All forms of continuing-existence occur currently}

With space itself, there is one form of continuing-existence, that of space. In a situation where matter is occupying space, there are two forms of continuing-existence, the continuing-existence of the matter, and the continuing-existence of the spatial-place the matter occupies. In a situation where matter is moving through space there are three forms of continuing-existence occurring together, that of the space the matter is moving through, that of the matter itself, and that of the ongoing existence of the motion of the matter through space.

The continuing-existence of the motion occurs concurrently with the continuingexistence of the matter that is moving. The continuing-existence of the motion and the continuing-existence of the matter both occur concurrently with the continuing-existence of the space through which the motion is passing - three concurrent forms of continuingexistence. 


\section{New parts of all forms of continuing-existence occur concurrently}

Because the three concurrent forms of continuing-existence, that of space, that of matter, and that of motion, are forms of ongoing noncoexistent-sequential-difference, each with the occurrence of new part, the occurrences of the new parts are concurrent.

\section{Existential-context role of spatial continuing-existence}

Just as space provides an extensional existential-context for the extensional qualities of matter and motion, spatial continuing existence provides a changing existential-context for the change that occurs with the continuing-existence of matter and the continuingexistence of motion.

Like spatial continuing-existence, all other forms of change occur as noncoexistentsequential-difference with initiation of new part. The noncoexistent-sequentialdifference, with occurrence of new part, of spatial continuing-existence constitutes an existential-context, a place to occur, for the noncoexistent-sequential-difference and the occurrence of new part of all other forms of change.

\section{Existential-dependency with continuing-existence}

Existential-dependency is a relation in which the existence and/or nature of one factor is dependent on the existence and/or role of another factor.

It is matter that moves. The continuing-existence of the motion is existentially-dependent on the continuing-existence of the moving matter. Both the matter and the motion exist in space. Space constitutes an existential-context, a place to be, for the matter and the motion. The continuing-existence of both the matter and the motion are existentiallydependent on the continuing-existence of the existential-context in which they exist. They are existentially-dependent on the continuing-existence of space.

The infinitely universal, omnipresent, ongoing change of spatial continuingexistence constitutes an existential-context for all the other forms of change.

- All other forms of change are limited in some manner.

- All other forms of change are existentially-dependent on spatial continuing-existence for a place to occur.

- All change occurs concurrently with the continuing-existence of the spatial-place where the change occurs.

- All change occurs currently with the continuing-existence of infinite space.

- To not occur concurrently with spatial continuing-existence is to not occur at all. 
As the continuing-existence of the foundational component of the universe, spatial continuing-existence is the foundational form of change in the universe. All change occurs concurrently with the continuing-existence of that foundation form of change.

\section{Spatial continuing-existence plays the role of time in the universe}

- The various qualities of time occur in the universe as qualities of the continuingexistence of the foundational component of the universe, as qualities of the continuing-existence of space.

- The manner in which the continuing-existence of space occurs establishes the qualities of time in the universe.

\section{Universal temporal change}

The continuing-existence of space occurs as a universal, infinitely omnipresent form of change - a continuously ongoing, smoothly sequential, unidirectional change, with continuous initiation of new part as space continues to exist.

Because space - infinite three-dimensional immaterial spatial-place - is the foundational component of the universe, and thereby constitutes an existential-context, a place to exist, for all else that exists, the continuing-existence of space constitutes an existential-context, a place to occur, for the continuing-existence of all that exists in space.

The continuing-existence of all that exists occurs concurrently with the continuingexistence of space.

All changes that occur to things that exist, occur during the continuing-existence of those things, and thus during the continuing-existence of the existential-context of their continuing-existence-during the continuing-existence of space.

All change occurs during and concurrent with the ongoing universal change of spatial continuing-existence.

The change that is spatial continuing-existence is here playing the role of universal temporal change.

\section{Establishing the Existence and the Intrinsic Nature of the Present}

With the continuously ongoing change of spatial continuing-existence only the current part exists. The prior part, that was occurring just previously, is no longer there. It has ceased to exist. And the following part has not yet occurred. It has not yet come into existence. 
With the continuing-existence of space only the current part exits-only the current part is present. The prior part is no longer occurring. The prior part was present, but is no longer present - it has passed. And the following part is a future part of spatial continuing-existence which does not yet exist, but will occur as new part of spatial continuing-existence.

- Space constitutes an existential-context for the existence of all else that exists.

- Spatial continuing-existence constitutes an existential-context for the continuingexistence of everything that exists in space, that is, for the continuing-existence of all else that exists.

- The currently present part of spatial continuing-existence constitutes an existential-context for the currently present part of the continuing-existence of everything that exists in space.

In this manner the currently present part of spatial continuing-existence is playing the role in the universe of the temporal present.

- Any ongoing change that happens to something that exists occurs during the ongoing continuing-existence of whatever it is that is changing.

- An ongoing change occurs concurrently with the continuing-existence of that which is changing.

- The present part of an ongoing change occurs concurrently with the present part of the continuing-existence of what is changing.

- An ongoing change and the ongoing continuing-existence of what is changing both occur concurrently with the existential-context for change and for continuing-existence provided by spatial continuing-existence.

- The currently present part of spatial continuing-existence provides an existentialcontext for the currently present part of an ongoing change and for the currently present part of the continuing-existence of what is changing.

The manner in which the continuing-existence of space occurs establishes the existence and the intrinsic nature of the temporal present.

\section{Identifying the Intrinsic Nature of the Past}

$>$ Only the current part of the continuing-existence of space exists — is present.

$>$ Only the current part of the continuing-existence of matter exists - is present.

$>$ Only the current part of ongoing change exists — is present.

* The prior part of ongoing change that was previously present, but is present no longer, does not exist - it has passed.

* The prior part of the continuing-existence of matter that was previously present, but is present no longer, does not exist - it has passed.

* The prior part of the continuing-existence of space that was previously present, but is present no longer, does not exist-it has passed. 
That which is no longer present does not exist-it has passed. The manner in which the continuing-existence of space occurs establishes the nature of the temporal past. It is what did occur as the present, but which no longer exists, having been replaced by the current present.

The term "the past" refers to the part of time that has gone before-to the prior part of spatial continuing-existence - the part that has passed. Because that which has passed does not exist, the term "the past" has no existing reality-referent. The concept has a conceptual-referent, but not a reality-referent.

Time travel to the past is not possible - there is nowhere to go.

\section{Identifying the Intrinsic Nature of the Future}

Space exists, and it continues to exist, with continuous new part of that ongoing existence. The current present determines by way of continuing-existence what will be. New part is initiated, progressively created, as the present by way of the ongoing continuing-existence of what has gone before. When the following part comes into existence by way of the initiation of new part of continuing-existence it does so as the current present.

The following part does not exist until it is initiated - it does not exist before it becomes the present. It never exists as the future distinct from or concurrent with its existence as the present. The manner in which the continuing-existence of space occurs establishes the nature of the temporal future - it does not exist - but it will. It is what will happen, but has not yet done so.

The term "the future" refers to the part of time that follows the present - to the following part of spatial continuing-existence - the part that has not yet occurred. Because the following part does not exist, the term "the future" has no existing reality-referent. The concept has a conceptual-referent, but not a reality-referent.

Because the future does not exist, time travel to the future is not possible - there is nowhere to go.

\section{Conclusion}

Space exists. It is there, and it continues to be there-space continues to exist.

The manner in which the continuing-existence of space occurs establishes time's arrow, and thereby the irreversibility of time, and additionally, the relentlessly ongoing quality of time. It establishes the existence and intrinsic nature of the present, and the relations of the present to the future and the past. 
The manner in which the continuing-existence of space occurs also establishes the universality and omnipresence of time such that a moment of time in any one place in space is the same moment throughout space. It also establishes that time is a form of change, and that that form of change is uniform in occurrence.

The manner in which the uniform change of the continuing-existence of space occurs establishes that time is evenly continuous, without any form of intrinsic units-a sequentially occurring nondimensional form of change with no shortest unit, no shortest period of time.

The continuing-existence of space - the occurrence of time-is independent of the existence of matter in any form. However, all forms of material change, including motion and the continuing-existence of matter itself, are existentially-dependent on the occurrence of the continuing-existence of space.

In the light of the knowledge that the continuing-existence of space plays the roles of time in the universe, the question now is what are the consequences for the philosophy of time, the practice of realist philosophy in general, and for the practice of the science of physics?

Smolin said, "Having begun my life in science searching for the equation beyond time, I now believe that the deepest secret of the universe is that its essence rests in how it unfolds moment by moment in time." [1]

\section{Bibliography}

1. Smolin L. Time Reborn. Boston, New York: Mariner Books, Houghton Mifflin Harcourt; 2014. pp. xi, xiii.

2. Vesterby V. The Identification of the Intrinsic Nature of Time. OSF Preprints [Preprint]. 2019 osf.io/gqxjy [posted 2019 Jul 26; revised 2019 Jul 29]: [17 p.]. Available from: https://doi.org/10.31219/osf.io/gqxjy doi: 10.31219/osf.io/gqxjy

3. Vesterby, Vincent. Emergence Is an Isomorphy. OSF Preprints [Preprint]. 2019 osf.io/ys3wd [posted 2019 Jul 4; revised 2019 Jul 9]: [7 p.]. Available from: https://doi.org/10.31219/osf.io/ys3wd doi:10.31219/osf.io/ys3wd

Here are two other papers that provide additional understanding about the intrinsic nature of time.

Vesterby V. Paul Davies and Why Time Is Not a Flow. OSF Preprints [Preprint]. 2019 osf.io/bh23c [posted 2019 Jul 27; revised 2019 Jul 29]: [7 p.]. Available from: https://doi.org/10.31219/osf.io/bh23c doi:-10.31219/osf.io/bh23c 
Vesterby V. Temporal Naturalism-Analysis of the Paper by Lee Smolin. OSF Preprints [Preprint]. 2019 osf.io/mk9wz [posted 2019 Jul 29; revised 2019 Jul 29]: [6 p.]. Available from: https://doi.org/10.31219/osf.io/mk9wz doi: 10.31219/osf.io/mk9wz 